\title{
Local Orientation Estimation in Corrupted Images
}

\author{
Franck MICHELET ${ }^{1}$, Jean-Pierre DA COSTA ${ }^{1}$, Pierre BAYLOU ${ }^{1}$, \\ Christian GERMAIN ${ }^{1}$ \\ ${ }^{1}$ LAPS - Signal \& Image Team \\ UMR N5131 CNRS - Bordeaux I University - ENSEIRB - ENITAB \\ 351, Cours de la Libération - 33405 Talence Cedex - France \\ christian.germain@laps.u-bordeaux1.fr
}

\begin{abstract}
IRON is a low level operator dedicated to the estimation of single and multiple local orientations in images. Previous works have shown that IRON is more accurate and more selective than Gabor and Steerable filters, for textures corrupted with Gaussian noise. In this paper, we propose two new features. The first one is dedicated to the estimation of orientation in images damaged by impulsive noise. The second one applies when images are corrupted with an amplitude modulation, such as an inhomogeneous lighting.
\end{abstract}

Keywords: Image Processing, Orientation estimation, Anisotropy, Impulse noise, Amplitude modulation, IRON.

\section{Introduction}

For three decades, many works have concerned orientation estimation in images. Applications of orientation estimation concern, for example, texture characterization [4][8], anisotropic diffusion [11][13] or image segmentation [2].

Orientations have specific characteristics which have to be taken into account in the estimation process. First, orientation doesn't always exist. In case of uniform grey level images or isotropic textures, no orientation can be estimated. Besides, when orientation exists, it depends on the scale of analysis. Considering that, generally, statistical techniques can be used to derive large scale orientation from local orientation [1][7][2], we will focus on local orientation estimation.

Differential approaches [5][8] are conventional for local orientation estimation. They are based on the local computation of first or second order derivatives of all the points of the image. Nevertheless these methods fail if more than one single orientation appear at a given location. In such a case, the response of derivative operators results from a non-linear mixture of the true local orientations.

Other popular methods for orientation estimation are based on a set of oriented filters. Among them, we can quote Gabor filters [2] [3] and Steerable filters [6] [10]. Operator IRON (Isotropic and Recursive Orientation Network) is another example of an oriented operator [9]. It consists in an oriented network of parallel lines along which we compute a homogeneity feature. The output of this feature indicates the confidence in the tested orientation. For such methods, accuracy and selectivity both depend on the number of filters and on the size of their computing support. Exercised 
on synthetic and real images, IRON provides more accuracy, noise robustness and selectivity than Gabor or Steerable filters [9].

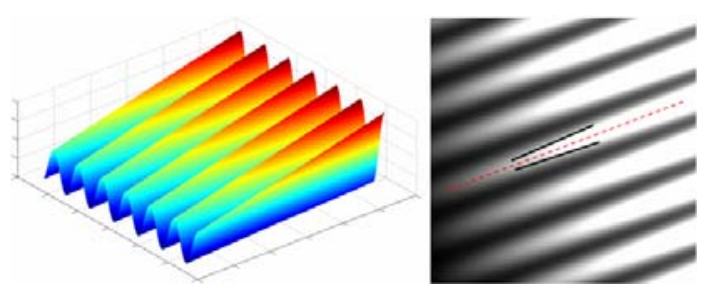

Fig. 1. Texture corrupted with amplitude modulation (profile function and grey level image). Isolevel lines orientation (solid lines), and original orientation (dashed line)

These methods are generally well suited for multiple local orientation estimation. Nevertheless, in some specific circumstances, they are unable to provide accurate and robust estimations. More particularly, we have found that when amplitude modulation occurs, orientation estimation becomes biased. Figure 1 shows a texture for which the sinusoidal profile is modulated with an affine function. This is the kind of images resulting, for example, from an inhomogeneous lighting. In this case, amplitude modulation affects the direction of isolevel curves which are not anymore equal to the perception of the orientation from the uncorrupted image. Therefore, all the classical orientation operators will provide us with an erroneous estimation.

When impulse noise occurs, classical operators also fail to estimate orientations properly. Figure 2a shows a directional texture corrupted with salt and pepper noise. Figure $2 b$ shows the local orientation estimation in this picture, using Gabor filters. The size of the computing support is equivalent the size of the arrows. Indeed, the salt and pepper noise affects significantly the orientation estimation. Other estimators such as the Steerable Filters or Gradient masks would provide even worse estimations at the same scale.

In this paper, we propose two new homogeneity features for IRON, in order to deal with each of these problems. The first one relies on the Robust Homogeneity Function $(R H F)$ instead of variance estimation, and will be effective in case of impulse noise.

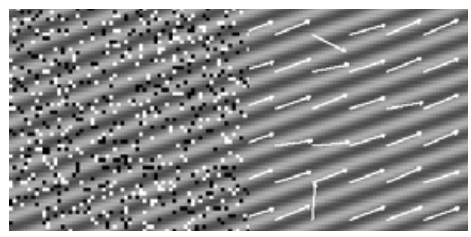

Fig. 2. Texture corrupted with salt and pepper noise and Gabor orientation estimation

The second one, based on the identification of local affine modulation parameters, solves the case of amplitude modulation.

In the second part of this paper, we shortly describe IRON, already introduced in [9]. In the third part, we propose two new homogeneity features. The first one is dedi- 
cated to images corrupted by impulse noise, and the second one to amplitude modulated directional textures. In the fourth part, we present and discuss some results.

\section{The IRON Orientation Estimator}

\subsection{General Presentation}

IRON was introduced in [9]. It is an oriented operator working in the spatial domain. Its principle is to compute a homogeneity feature along a network of parallel lines oriented $\theta$. This feature depends on the grey levels of the pixels found on these lines.

Each network is made of $L$ lines and each line consists in $p$ points. The distance between each line and the distance between two consecutive points on a line are equal to the pitch of the pixel grid.

The network can be either symmetric or asymmetric. In the first case, the lines lie on both sides of the central point. The resulting orientation is estimated modulo $\pi$. In the second case, the lines lie only on one side of the central point thus providing with an orientation modulo $2 \pi$.

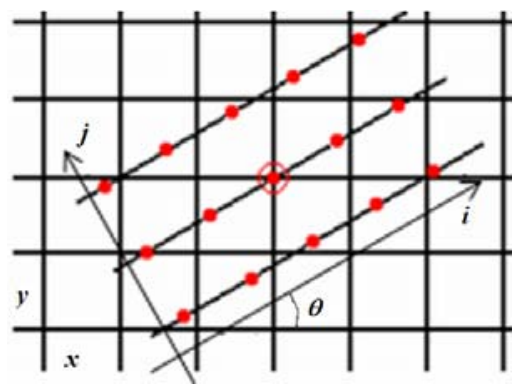

Fig. 3. IRON symmetric network of 3 lines and 5 points per line.

Since the network points do not always line up on the pixel grid, the grey level values of the network points (Fig. 3) are computed using a bi-dimensional interpolation.

In [9], we have proposed an implementation based on the rotation of the image instead of the rotation of the network itself. This implementation reduces the computational cost of the interpolation stage.

\subsection{Network Tuning}

The parameters $L$ and $p$ act upon both the size and the shape of the network.

The shape of the network affects its selectivity and also its noise robustness. The size of the network depends on the scale of analysis. Increasing the number of lines allows increasing noise robustness. However, in the same time the selectivity of our operator decreases. 
Another important aspect of IRON is the choice of the homogeneity feature. We have already proposed in [9] the following homogeneity feature $H$.

$$
H(x, y, \theta)=\left(\varepsilon_{0}+\sum_{j=1}^{L} \sum_{i=1}^{p-1}\left|v_{i, j, \theta}-v_{i+1, j, \theta}\right|\right)^{-1}
$$

$v_{i, j, \theta}$ is the interpolated grey level on the $i^{\text {th }}$ point from the $j^{\text {th }}$ line of the network oriented $\theta$ (Fig. 3). $\varepsilon_{0}$ is a constant close to 0 . It avoids the denominator to be null.

In the general case of an image corrupted with a Gaussian noise, the most appropriate function relies on variance estimation.

$$
V(x, y, \theta)=\left(\varepsilon_{0}+\frac{1}{L(p-1)} \sum_{j=1}^{L} \sum_{i=1}^{p}\left(v_{i, j, \theta}-\frac{1}{p} \sum_{k=1}^{p} v_{k, j, \theta}\right)^{2}\right)^{-\frac{1}{2}}
$$

For both features, the recursive implementation described in [9] is possible, thus reducing considerably its computational cost.

In case of more complex textures, for instance, when amplitude modulation or impulse noise occurs, other features can be defined in order to be more suited to the local configuration.

\section{New Features for IRON}

\subsection{Robust Homogeneity Function}

The classical homogeneity feature for IRON is based on variance estimation, and then it is more appropriate in case of a Gaussian noise. We propose here a new feature specifically designed to tackle impulse noise.

This new feature relies on a robust estimation of the homogeneity, using two medians instead of the two means in (2). It consists in computing along each line of the network "the median of the deviation from the median grey level".

For a network of $L$ lines and $p$ points per line, with orientation $\theta$, we obtain the Robust Homogeneity Function RHF:

$$
\operatorname{RHF}(x, y, \theta)=\left(\varepsilon_{0}+\frac{1}{L} \sum_{j=1}^{L} M_{i=1}^{p}\left(\left|v_{i, j, \theta}-M_{k=1}^{p}\left(v_{k, j, \theta}\right)\right|\right)\right)^{-1}
$$

where $\mathrm{M}($.$) stands for the median operator and v_{i, j, \theta}$ for the grey level of a point of the network.

Since the value of the RHF feature does not depend on the extreme values of the grey levels found on the network, it will be robust to a noise strongly corrupting a small number of pixels. 


\subsection{Affine Model Identification}

We propose now a second feature for IRON. Its aim is to provide with unbiased orientation estimations when amplitude modulation affects the directional texture. Let consider a horizontal directional texture, corresponding to the following intensity model:

$$
\hat{f}(i, j)=h(j) \cdot g(i)
$$

where $h(j)$ is a the profile function, and $g(i)$ the modulation function.

In order to estimate the orientation $\hat{\theta}$ with IRON, we design a feature which minimizes the quadratic difference $\varepsilon$ between the intensity $f(i, j)$ and the model:

$$
\varepsilon(\theta)=\sum_{(i, j) \in V(\theta)}(h(j) \cdot g(i)-f(i, j))^{2}
$$

$V(\theta)$ is the neighborhood used to compute IRON in the direction $\theta . L$, the number of lines and $p$, and the number of points per line define the dimensions of this neighborhood.

Let us consider that the modulation is slow compared with the variation of the profile function $h$. Therefore, this modulation can be assumed to be locally linear and $g(i)$ can be approximated by an affine function: $g(i)=1+\alpha . i$.

The quadratic difference then becomes:

$$
\varepsilon(\theta)=\sum_{i=1}^{p} \sum_{j=1}^{L}(h(j)(1+\alpha . i)-f(i, j))^{2}
$$

The minimum value for $\varepsilon$ is obtained when its derivatives, regarding $h$ and $\alpha$, are null.

$$
\frac{\partial \varepsilon}{\partial h}=2 \sum_{i=1}^{p} \sum_{j=1}^{L}(1+\alpha . i) \cdot(h(j)(1+\alpha . i)-f(i, j))=0
$$

and

$$
\frac{\partial \varepsilon}{\partial \alpha}=2 \sum_{i=1}^{p} \sum_{j=1}^{L} i \cdot h(j) \cdot(h(j)(1+\alpha . i)-f(i, j))=0
$$

From these equations we obtain:

$$
h(j)=\frac{\sum_{i=1}^{p} f(i, j)+\alpha \sum_{i=1}^{p} i \cdot f(i, j)}{p+\alpha \cdot p \cdot(p+1)+\alpha^{2} \frac{p \cdot(p+1) \cdot(2 p+1)}{6}}
$$

and 


$$
\alpha=\frac{\sum_{j=1}^{L} \sum_{i=1}^{p} h(j) \cdot i \cdot f(i, j)-\frac{p \cdot(p+1)}{2} \sum_{j=1}^{L} h(j)^{2}}{\frac{p \cdot(p+1) \cdot(2 p+1)}{6} \sum_{j=1}^{L} h(j)^{2}}
$$

Let us define $K_{1}, K_{2}, K_{3}$ :

$$
K_{1}=\sum_{j=1}^{L}\left(\sum_{i=1}^{p} f(i, j) \cdot \sum_{i=1}^{p} i \cdot f(i, j)\right) K_{2}=\sum_{j=1}^{L}\left(\sum_{i=1}^{p} f(i, j)\right)^{2} K_{3}=\sum_{j=1}^{L}\left(\sum_{i=1}^{p} i \cdot f(i, j)\right)^{2}
$$

Introducing the following terms in (10), we finally obtain:

$$
\begin{aligned}
& \alpha^{2}\left(K_{1}(p+1)(2 p+1)-3 K_{3}(p+1)\right) \\
& +\alpha\left(K_{2}(p+1)(2 p+1)-6 K_{3}\right)+3 K_{2}(p+1)-6 K_{1}=0
\end{aligned}
$$

Solving this equation allows us to determine the affine modulation function $g$ and profile function $f$ for each of the tested orientations. The minimum value of $\varepsilon(\theta)$ indicates the orientation for which the model fits the best with the image.

As this framework has been designed using an affine modulation model, it will apply perfectly for a texture affected by an illumination gradient (Fig. 1). We will see in the result section that it is also effective in case of a non affine modulation, while this modulation is slow regarding the amplitude variations of the profile function.

\section{Results and Discussion}

\subsection{Impulse Noise}

In order to compare the efficiency of various orientation estimators, we use synthetic textures corrupted by a salt and pepper noise. However, any kind of impulse noise could be considered as well. The profile function of the synthetic texture is a sine with period 6 pixels and $\theta=20^{\circ}$ (Fig. 2). For each operator we compute the Mean Angular Deviation $(M A D)$ in order to depict the effect on the noise on the orientation estimation.

$$
M A D=\frac{1}{N} \sum_{(x, y)} \Delta(\hat{\Theta}(x, y), \Theta(x, y))
$$

where $N$ is the size of the sample (i.e. the number of pixels $(x, y)$ considered), $\hat{\Theta}$ stands for the estimated orientation and $\Delta\left(\theta_{1}, \theta_{2}\right)=\min \left(\left|\theta_{1}-\theta_{2}\right|, \pi-\left|\theta_{1}-\theta_{2}\right|\right), \theta \in[0, \pi[$.

We compute IRON symmetric with the RHF feature and compare the results with Gabor (quadrature) filters [2] [3] and Steerable (E4) filters [6] [10]. We test the following sizes for the computing support 11x11 and 21x21. All other parameters for Gabor and Steerable Filters are tuned in order to get the best estimations. In each case, 
180 orientations are tested (angular step $=1^{\circ}$ ) for 100 noise realizations, giving the following results.

Table 1. Angular error $M A D$ for synthetic textures (sine profile function with period 6 pixels and $\theta=20^{\circ}$ ), corrupted with impulse noise

\begin{tabular}{|c|c|c|c|}
\hline $\begin{array}{c}M A D \\
(\text { degrees })\end{array}$ & $\begin{array}{c}\text { Computing } \\
\text { Support Size }\end{array}$ & $\begin{array}{c}\text { Noisy Pixels: } \\
5 \%\end{array}$ & $\begin{array}{c}\text { Noisy Pixels: } \\
20 \%\end{array}$ \\
\hline \multirow{2}{*}{$\begin{array}{c}\text { IRON } \\
(R H F)\end{array}$} & $11 \times 11$ & $0.6^{\circ}$ & $4.0^{\circ}$ \\
\cline { 2 - 4 } & $21 \times 21$ & $0.0^{\circ}$ & $0.7^{\circ}$ \\
\hline \multirow{2}{*}{ Gabor } & $11 \times 11$ & $16.0^{\circ}$ & $24.0^{\circ}$ \\
\cline { 2 - 4 } & $21 \times 21$ & $0.6^{\circ}$ & $1.6^{\circ}$ \\
\hline \multirow{2}{*}{$\begin{array}{c}\text { Steerable } \\
(E 4)\end{array}$} & $11 \times 11$ & $3.4^{\circ}$ & $0.9^{\circ}$ \\
\cline { 2 - 4 } & $21 \times 21$ & $14.2^{\circ}$ & $2.7^{\circ}$ \\
\hline
\end{tabular}

Using the feature $R H F$ with IRON gives the best estimations whatever the support size or the ratio of noisy pixels. Experiments with other textures, noises or computing support size confirm these results.

Figure 4 shows the results obtained with a real fingerprint image corrupted with impulse noise. The noisy pixel ratio is $20 \%$. For all filters, computing support size is $15 \times 15$. This size is a fair compromise in order to obtain smooth orientation maps and to detect minutiae. All other parameters for Gabor and Steerable Filters are tuned in order to get the best estimations. 180 orientations are tested (angular step $=1^{\circ}$ ).

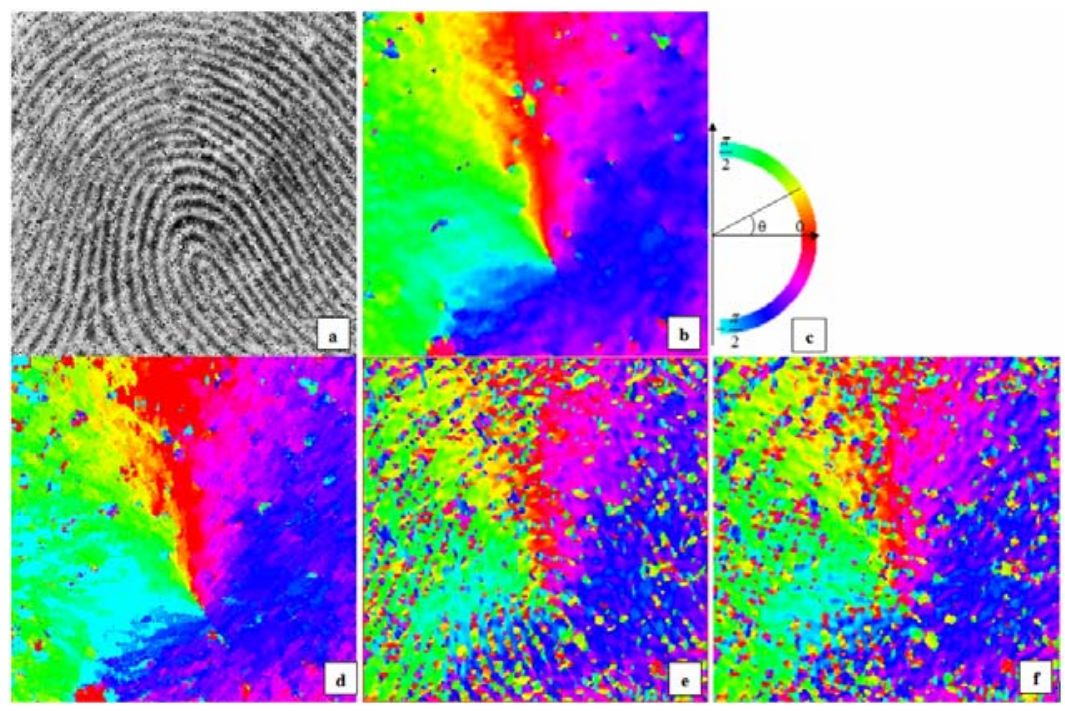

Fig. 4. a: Fingerprint image corrupted with $20 \%$ impulse noise; b: Orientation map without noise (IRON Variance); c: Color palette; d: Orientation map using IRON RHF; e: Orientation map using Gabor filters; f: Orientation map using Steerable E4 filters. 
Figure $4 \mathrm{~b}$ is the reference orientation map, computed applying IRON with its original variance feature [9] to the uncorrupted version of Figure 4a. The map is smooth everywhere except around minutiae.

Figure 4d shows that the results obtained with IRON $R F H$ on the corrupted image are very close to the reference map even if some errors appear.

On the opposite, Figure 4e and $4 \mathrm{f}$ show that Gabor and the Steerable filters are strongly affected by the impulse noise. Theses maps are very irregular and estimation error close to $90^{\circ}$ are frequent.

\subsection{Amplitude Modulation}

For this experiment, we exercise IRON with affine model identification for three kinds of synthetic textures. The profile function of theses textures is a sine with period 10 pixels with various orientations. The first texture, called Tex1, is corrupted using an affine modulation with the same orientation as the texture in Fig. 1. Tex2 is corrupted using the same affine modulation but with a different orientation. Tex3 is corrupted using a non affine modulation: $g(i)=1+A_{\bmod } \cdot \sin \left(2 \pi \cdot i / T_{\bmod }\right)$ with $T_{\bmod }=50$ pixels and $A_{\text {mod }}=0.5$ (Fig 4a).

Table 2 shows the MAD values obtained with IRON, Gabor (quadrature) and Steerable Filters E4.

Table 2. Angular error $M A D$ in case of amplitude modulation

\begin{tabular}{|c|c|c|c|}
\hline $\begin{array}{c}M A D \\
\text { (degrees) }\end{array}$ & $\begin{array}{c}\text { IRON } \\
\text { (Affine) }\end{array}$ & $\begin{array}{c}\text { Steerable } \\
(\mathrm{E} 4)\end{array}$ & Gabor \\
\hline Tex1 & $0.0^{\circ}$ & $1.03^{\circ}$ & $0.28^{\circ}$ \\
\hline Tex2 & $0.0^{\circ}$ & $1.01^{\circ}$ & $0.22^{\circ}$ \\
\hline Tex3 & $0.0^{\circ}$ & $1.08^{\circ}$ & $7.23^{\circ}$ \\
\hline
\end{tabular}
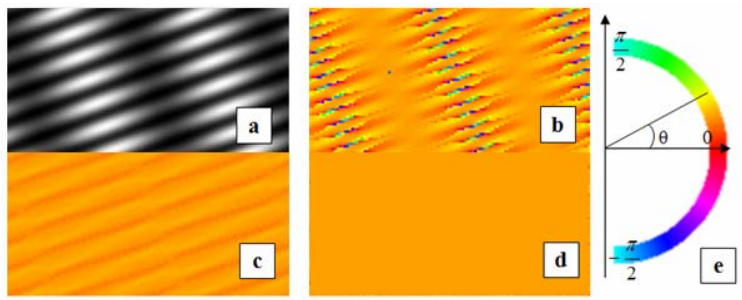

Fig. 5. a: Texture (Tex3) (non affine amplitude modulation); b: Gabor Filters; c: Steerable Filters (E4); d: IRON Affine; e: Orientation palette

Unlike Gabor and Steerable filters, our new feature appears to be insensitive to amplitude modulation, even in case of a non affine modulation (Fig. $5 d$ ).

Figure 6 shows the results obtained with an ancient engraving image corrupted with non affine amplitude modulation. The period of the modulation is 30 pixels, and 
its orientation is $30^{\circ}$. For all filters, computing support size is set to $15 \times 15$. All other parameters for Gabor and Steerable Filters are tuned in order to get the best estimations. 180 orientations are tested (angular step $=1^{\circ}$ ).

Figure $6 \mathrm{~b}$ is the reference orientation map. It is computed applying the classical IRON variance feature [9] to the uncorrupted version of figure 6a. Figure 6d depicts the results obtained with IRON Affine. As expected, the amplitude modulation does not significantly affect the corrupted image.

On an another hand, Figure 6e and 6f show that Gabor and the Steerable filters are strongly influenced by the modulation, even for the thin vertical lines.

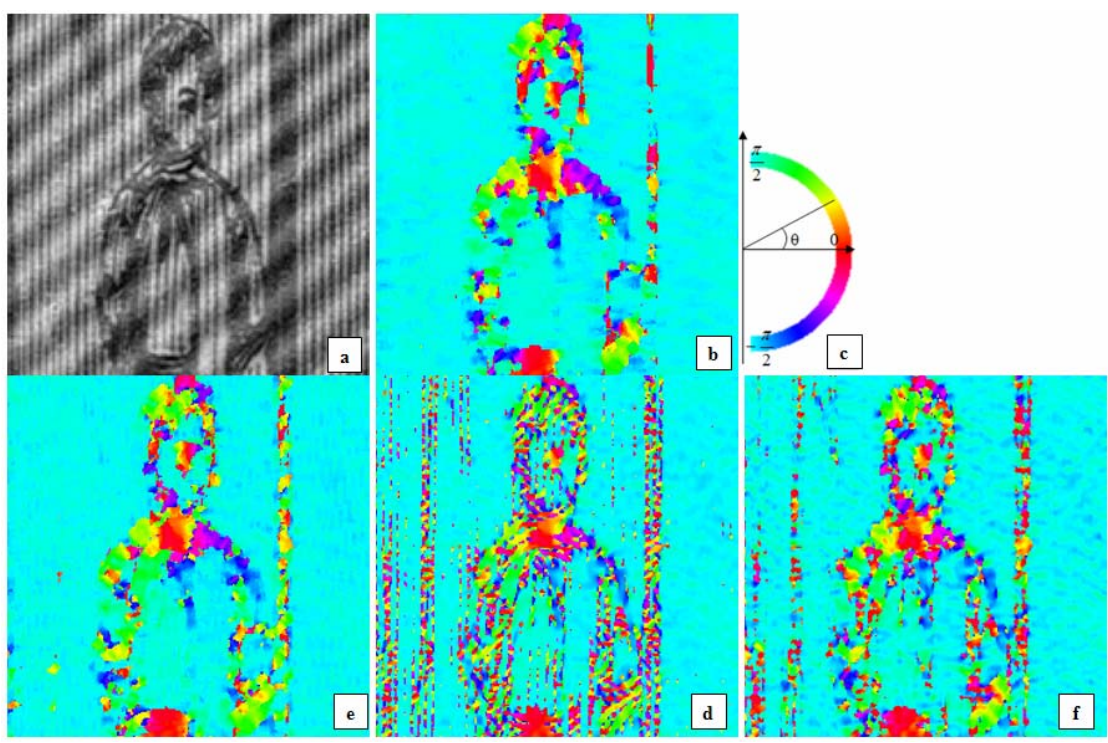

Fig. 6. a: Engraving image corrupted with amplitude modulation; b: Orientation map without noise (IRON Variance); c: Color palette; d: Orientation map using IRON Affine; e: Orientation map using Gabor filters; f: Orientation map using Steerable E4 filters.

\section{Conclusion}

IRON is a general framework for single and multiple local orientation estimation. Previous works have shown that IRON is more accurate and selective than classical operators, for textures corrupted with Gaussian noise.

In this paper, we have introduced two new homogeneity features which allow us to adapt IRON when impulsive noise or amplitude modulation occurs. Exercised on both synthetic and real images, these new features show their efficiency to overcome such perturbations.

Therefore, knowing a priori the kind of perturbation which corrupts the image allows us to choose the appropriate feature and thus enhance the adaptability of the IRON network for single and multiple local orientation estimation. 
Acknowledgements. Let us thank the FEDER InterReg IIIB (PIMHAI project) for its financial support.

\section{References}

1. Bigün, J., Bigün, T., Nilsson, K.: Recognition by symmetry derivatives and the generalized structure tensor, IEEE Transactions on PAMI, Vol. 26, no.12, (2004) 1590-1605.

2. Bigün, J., du Buf, J.H.: N-folded symmetries by complex moments in Gabor space and their application to unsupervised texture segmentation, IEEE Trans. on PAMI, Vol. 16, no. 1. (1994) 80-87.

3. Chen, J., Sato, Y., Tamura, S.: Orientation Space Filtering for Multiple Line Segmentation, Proc. of IEEE Conference on Computer Vision and Pattern Recognition, California, (1998).

4. Chetverikov, D., Hanbury, A.: Finding defects in texture using regularity and local orientation, Pattern Recognition, Vol. 35. (2002) 2165-2180.

5. Deriche, R.: Fast Algorithms for Low-Level Vision, IEEE Transactions on PAMI, Vol. 12, no.1. (1990) 78-81.

6. Freeman, W.T., Adelson, E.H.: The design and use of steerable filters, IEEE Trans. on PAMI, Vol. 13, no.9. (1991) 891-906.

7. Knutsson, H.: Representing Local Structure Using Tensors, Proceedings of Scandinavian Conference on Image Analysis, Oulu, Finland, (1989).

8. Le Pouliquen, F., Da Costa, J.P., Germain, Ch., Baylou P.: A new adaptive framework for unbiased orientation estimation, Pattern Recognition, Vol. 38. (2005) 2032-2046.

9. Michelet F., Germain Ch., Baylou P., Local Multiple Orientation Estimation: Isotropic and Recursive Oriented Network, Proc. of ICPR 2004, Cambridge, UK, (2004).

10. Perona, P.: Deformable kernels for early vision, IEEE Transactions on PAMI, Vol. 17, no.5. (1995) 488-499.

11. Perona, P., Malik, J.: Scale-space and edge detection using anisotropic diffusion, PAMI Vol. 12, no. 7. (1990) 629-639.

12. Rao, A.R.: A Taxonomy for Texture Description and Identification, Springer, (1990).

13. Terebes, R., Lavialle, O., Baylou, P., Borda, M.: Orientation driven diffusion, Acta Technica Napocensis-Electronics and Telecommunications, Cluj-Napoca, Vol 42, no.2, (2002) 20-24. 